\title{
Comprehensive Evaluation of the IEEE 802.15.4 MAC Layer Performance with Retransmissions
}

\author{
A. Faridi, M. R. Palattella, A. Lozano, M. Dohler, G. Boggia, A. Grieco, and P. Camarda
}

\begin{abstract}
Supported by IEEE 802.15.4 standardization activities, embedded networks have been gaining in popularity in recent years. The focus of this paper is to quantify the behavior of key networking metrics of IEEE 802.15.4 beacon-enabled nodes under typical operating conditions, with the inclusion of packet retransmissions. We correct and extend previous analyses by scrutinizing the assumptions on which the prevalent Markovian modeling is generally based on. This allows us to rigorously derive key metrics such as throughput, delay, power consumption, and packet discard probabilities. We finally show by means of a comparative study the superior accuracy of our modeling approach.
\end{abstract}

\section{INTRODUCTION}

ZigBee [1] is arguably the most prominent alliance dedicated to low-power embedded systems. It is a facilitator of applications pertaining to home and building automation, smart metering, sensing, and health care, among many others. Its link and access protocols rely on the specifications of IEEE 802.15.4 [2], whereas higher layers are subject to the profile definition of the ZigBee special interest group. ZigBee is gaining in importance and the underlying IEEE standard ensures that technology is available from multiple vendors. On the downside, true deployment success stories are fairly rare still, possibly due to the fact that it operates in the 2.4$\mathrm{GHz}$ ISM band where it suffers from inter- and intra-system interference.

This paper is precisely focused on the intra-system behavior in that we aim to quantify the performance of the IEEE 802.15.4 medium access control (MAC) protocol, crucial in a successful system deployment with multiple parties suffering from contention. IEEE 802.15.4 enjoys two channel-access methods, namely, the non-beacon mode for low traffic and the beacon-enabled mode for medium and high traffic. The former uses standard carrier sensing multiple access (CSMA) for conflict resolution and positive acknowledgments for successfully received packets; since CSMA per se has already been well covered in the open literature, it will not be further investigated. The latter is a flexible approach able to mimic the behavior of a large set of previously published wireless sensor network MACs such as framed MACs, contentionbased MACs with common active periods, sampling protocols with low duty cycles, and hybrids thereof [3]; it follows a flexible superframe structure where the network coordinator

A. Faridi and A. Lozano are with the Department of Information and Communication Technologies at Universitat Pompeu Fabra, Spain. M. R. Palattella, G. Boggoa, A. Grieco, and P. Camarda are with Dipartimento di Elettrotecnica ed Elettronica, Politecnico di Bari, Italy. Mischa Dohler is with the Centre Tecnolgic de Telecomunicacions de Catalunya (CTTC), Spain transmits beacons at predetermined intervals. It successfully combats the main sources of energy drainage by minimizing idle listening, overhearing, collisions and protocol overheads, thereby covering a large number of envisaged ZigBee applications sufficiently well.

The behavior of the slotted CSMA/CA in an IEEE 802.15.4 network has often been investigated using the approach introduced by Bianchi [4] for the IEEE 802.11 CSMA/CA, based on modeling a single node's behavior with a discretetime Markov chain. In these models, the state of each node evolves through its corresponding Markov chain independently of other nodes' states except for when it is sensing the channel.

In [5], Mišić et al. propose a Markov chain model to analyze the slotted CSMA/CA under saturated and unsaturated traffic conditions in a beacon-enabled network in acknowledgement (ACK) mode; the superframe structure and the retransmissions are not considered. A basic per-node Markov chain is constructed for the saturated case, although with some states missing in both the transmission and ACK-awaiting stages. This model is then extended to the saturated case. In [6], the unsaturated model of [5] is further extended to include also the superframe structure and retransmissions. Since the unsaturated models in both [5] and [6] are extensions of the saturated Markov chain model in [5], the aforementioned issues apply to them as well.

Pollin et al. [7] suggest a Markov chain model for the slotted CSMA/CA under saturated and unsaturated periodic traffic conditions in a beacon-enabled network in both ACK and no-ACK modes. This model is simple but, in contrast with [5] and [6], it is complete. The superframe structure is not modeled. The model in [7] relies on the assumption that the probability to start sensing the channel is independent across the nodes, and it properly considers the dependence between the nodes when calculating the channel occupancy probabilities. However, as detailed subsequently, the model suffers from a number of shortcomings.

Other works using similar approaches to Pollin and Mišić include [8] and [9]; however, similar issues as the ones just mentioned can be observed in their models and calculations as well. In more recent works, Wen et al. [10] use a proper Markov chain model, but they do not consider the dependence in transmission probability of different nodes when calculating the sensing probabilities. Finally, in [11], Jung et al. propose a new model based on the Mišić model in [6]. However, they too do not include all the transmission states in the Markov chain.

With respect to the above prior art, the core contribution of this paper is the calculation of all the network metrics 
(throughput, delay, power consumption, etc) necessary for a system engineer to correctly dimension the network. It is based on the following advancements:

- We examine the assumptions that underlie the traditional Markov chain analyses, which is unprecedented and which allows identifying some shortcomings of the prior art and pave the way for a novel approach.

- Scrutinizing the prior art, we identify imprecise uses of the Markov chain approach, embodied by the presence of extra states and by the inexact definition of the packet failure probability, among others.

- This stimulates us to redefine various parameters which, against prior assumptions, are conditioned on other events taking place in the network.

- All this allows us to correctly and rigorously consider retransmissions, with the inclusion of acknowledgment states and with the recognition that successive transmission attempts for a given packet experience distinct success probabilities.

As part of this work, a comprehensive event-driven simulator has been assembled in order to validate the analytical derivations and to aid in the computation of some of the quantities of interest. This simulator, detailed in Appendix D, is freely available to the research community.

The paper is organized as follows. In Section II, we briefly detail the IEEE 802.15.4 MAC protocol so as to aid the understanding of subsequent sections. In Section III, we detail the Markov chain and introduce the basic notation. Section IV is then dedicated to the underlying system assumptions and a rigorous scrutiny of these assumptions, their applicability and limitations. In Sections V and VI, we then calculate the aforementioned network performance metrics respectively using the traditional as well as loosened Markov chain assumptions. Finally, Section VII concludes the paper.

\section{IEEE 802.15.4 MAC PROTOCOL DESCRIPTION}

In a Low Rate Wireless Personal Area Network (LRWPAN), the PAN coordinator (i.e., the central controller) builds the network in its personal operating space. Communications from nodes to coordinator (uplink), from coordinator to nodes (downlink), or from node to node (ad hoc) are possible.

In the beacon-enabled mode, which is the object of our attention, the time axis is structured as a sequence of superframes bounded by beacon frames. Each superframe comprises:

- An active part made up of a contention access period (CAP) and an optional contention free period (CFP). During the CAP, nodes access the channel using slotted CSMA/CA. The optional CFP is activated upon request from the nodes to the PAN coordinator for allocating guaranteed time slots.

- An optional inactive part where the devices do not interact with the PAN coordinator and could enter a low-power state to save energy.

The slotted CSMA/CA mechanism adopted with the beacon-enabled mode of IEEE 802.15.4 is different from the well-known IEEE 802.11 CSMA/CA scheme [12]. The main differences involve the time slotted behavior, the backoff algorithm, and the clear channel assessment (CCA) procedure used to sense whether the channel is idle. Specifically:

- In IEEE 802.15.4, each operation (channel access, backoff count, CCA) can only begin at the boundary of time slots, which recall are termed backoff periods. In IEEE 802.11 , the notion of a slot exists only insofar as backoff counting is concerned.

- In IEEE 802.15.4, only when the backoff counter reaches zero does the node sense the channel (CCA). In IEEE 802.11, nodes are constantly sensing while in backoff, thereby incurring an additional consumption of energy.

- In IEEE 802.15.4, the backoff counter of a node decreases regardless of whether the channel is idle or busy. In contrast, in IEEE 802.11 the backoff counting pauses whenever the channel becomes busy.

The basic time unit of the IEEE 802.15.4 MAC protocol is the backoff period, a time slot of length aUnitBackoffPeriod.

For each generated packet, each node maintains a variable $r$ indicating the number collisions that the packet has undergone. ${ }^{1}$ For a newly generated packet, $r$ is initialized to 0 . Moreover, for each transmission attempt, the node maintains three variables: $N B, C W$, and $B E$. $N B$ indicates the backoff stage or, equivalently, the number of times the backoff procedure has been repeated while attempting the current transmission. $C W$ is the contention window length, which defines the number of backoff periods the channel has to be sensed idle before transmission can start. $B E$ is the backoff exponent used to draw the random backoff value and it should satisfy macMinBE $\leq B E \leq \operatorname{macMaxBE}$, where macMinBE and macMaxBE are constants.

The slotted CSMA/CA mechanism works as shown in Fig. 1. Before every new transmission, $N B, C W$ and $B E$ are initialized to 0,2 , and $\operatorname{macMin} B E$, respectively. The node waits for a random number of backoff periods specified by the backoff value, drawn uniformly in the range $\left[0,2^{B E}-1\right]$. Then, it performs the first CCA, i.e., it senses the channel and, if it is idle, the first CCA succeeds and $C W$ is decreased by one. The node then performs the second CCA and, if that is also successful, it can transmit the packet.

If either of the CCAs fail, both $N B$ and $B E$ are incremented by one, ensuring that $B E$ levels at $\operatorname{macMaxBE}$, and $C W$ is reset to 2 . The node repeats the procedure for the new backoff stage by drawing a new backoff value, unless the value of $N B$ has become greater than a constant $M=$ macMaxCSMABackoffs. In that case, the CSMA/CA algorithm terminates with a channel access failure status and the concerned packet is discarded.

The network can be operating in either acknowledged or unacknowledged modes. Hereafter, we refer to these modes as the ACK mode and the no-ACK mode, respectively. In both modes, a packet transmitted after a successful channel access procedure can either be received successfully or experience a collision. In the no-ACK mode, regardless of

\footnotetext{
${ }^{1}$ We identify all unsuccessful transmissions with collisions, focusing on systems where interference among nodes is the dominant source of transmission failures and the effects of fading or noise are secondary.
} 


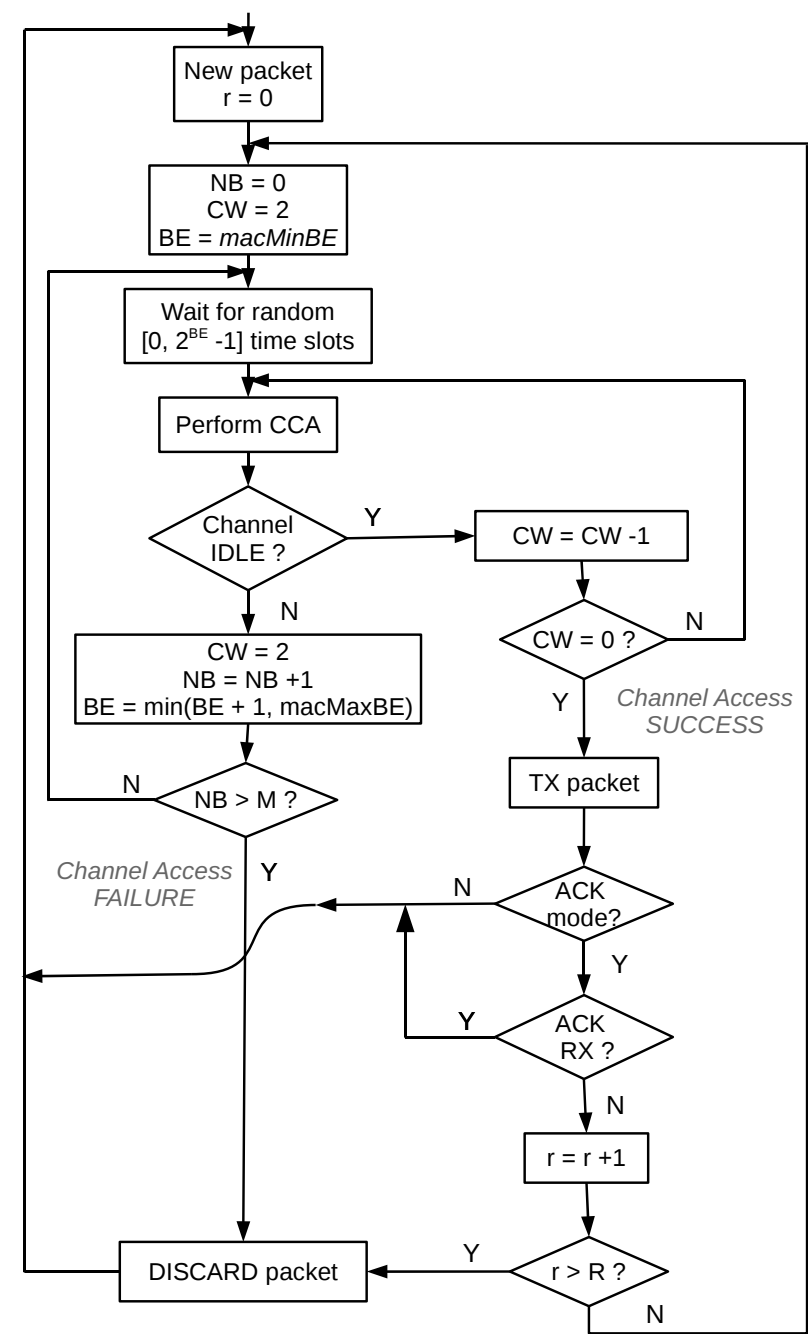

Fig. 1. Flow chart of the channel access procedure.

whether the transmission is successful or ends in collision, no acknowledgment will be received by the sending node and thus the node immediately continues the CSMA/CA procedure on a new packet. In the ACK mode, a successful transmission is accompanied by the reception of an ACK, which has a fixed length of $L_{a c k}$ backoff periods. The ACK is fed back to the sender node after a minimum time which allows for the transceiver of the node to switch from transmission mode (TX) to reception mode (RX). The ACK is expected by the sender node to be received before a fixed maximum time of macAckWaitDuration. If the ACK is not correctly received after this time, a collision is declared. A collided packet can be retransmitted at most $R=$ aMaxFrameRetries times if required, before being discarded. Therefore, every time a transmitted packet collides, $r$ is incremented by 1 , and if $r \leq R$, the packet is retransmitted using a new transmission procedure with $N B, C W$, and $B E$ reset to their initial values.

\section{Model AND NotATION}

Our Markov chain model, as well as our notation, closely follow those introduced in [7] with some necessary modifications, both to include retransmissions and to improve the accuracy of the model as will be described in due course.

\section{A. Markov Chain Model}

In [7], the performance of a single-hop LR-WPAN, composed by $N$ nodes and a PAN coordinator, is evaluated for uplink traffic. Saturated traffic conditions (i.e., when each of the $N$ nodes always has a packet available for transmission), under ACK and no-ACK modes, are considered. ${ }^{2}$ Here, we focus on the saturated case in ACK mode with retransmissions. The ACK-mode was extensively studied in [13]. We further focus our attention on the CAP, which is the most interesting superframe period from a MAC perspective. To that end, we do not explicitly consider the superframe structure and simply model an infinitely long CAP. We also assume that all data packets have the same length ${ }^{3}$ and that their transmission takes an integer multiple of the backoff period.

In [7], the ACK mode is considered but without collided packets being retransmitted. In our work, we do consider retransmissions, thereby obtaining a complete model of the behavior of a system in ACK mode. Fig. 2 shows the outline of the Markov chain model for the behavior of a node in ACK mode with retransmissions. We assume that the behavior of a given transmission attempt is the same regardless of the number of unsuccessful previous attempts on the current packet. In other words, Fig. 2 consists of $R+1$ replicas of the Markov chain modeling a single attempt. This assumption is more precisely stated in Section IV as assumption A2, and is used in Section V to calculate various network metrics. As we will see in Section VI, this assumption does not affect the accuracy of most of the calculated metrics. However, when calculating the average delay, it leads to some inaccuracy. We offer an alternative way of calculating the average delay without the use of this assumption in Section VI.

Given the aforementioned assumption, the behavior of the network can be analyzed by looking only at the per-attempt Markov chain model of a node. In detail, the behavior of a single node per-attempt is modeled using a two-dimensional Markov chain, with states represented by $\{s(t), c(t)\}$ at a given backoff period $t$, as shown in Fig. 3. Hereafter, we use the term slot to refer to a backoff period. All events happen at the beginning of a slot.

At a given slot $t$, the stochastic process $s(t)$ represents the transmission stage when $s(t)=-1$, the ACK stage when $s(t)=-2$, and the backoff and CCA stages when $s(t) \in$ $\{0, \ldots, M\}$. Depending on the value of $s(t)$, the stochastic process $c(t)$ acquires a different significance as follows:

- When the node is in transmission (i.e., $s(t)=-1), c(t) \in$ $\{0, \ldots, L-1\}$ represents the state of packet transmission, i.e., the number of slots spent thus far on the current transmission. $L$ is the packet size, measured in number of slots and including the overhead introduced by the PHY and MAC headers.

- When the node is in the ACK states (i.e., $s(t)=-2$ ), $c(t)=0$ represents the state used for switching the transceiver from TX to RX. Since the packet length is

\footnotetext{
${ }^{2}$ The saturated case reflects a sensor network scenario in which an event is detected by many nodes that want to transmit the gathered information at the same time.

${ }^{3}$ Again, this is consistent with a scenario where multiple nodes report the same event.
} 
TABLE I

MARKOV CHAIN STATES

\begin{tabular}{c|c||c|c|c|c|c|c|}
\multicolumn{2}{c||}{} & \multicolumn{6}{c|}{$c(t)$} \\
\cline { 2 - 8 } \multicolumn{2}{c||}{} & -2 & -1 & 0 & 1,2 & $3 \cdots L$ & $L+1 \cdots W_{M}-1$ \\
\hline \hline \multirow{3}{*}{$s(t)$} & $0 \cdots M$ & - & $\mathrm{CCA}_{2}$ & $\mathrm{CCA}_{1}$ & \multicolumn{4}{c|}{ Backoff } \\
\cline { 2 - 8 } & -1 & - & - & \multicolumn{2}{|c|}{ Packet Transmission } & - \\
\cline { 2 - 8 } & -2 & ACK No RX & ACK Idle Wait & ACK RX & - & - \\
\hline
\end{tabular}

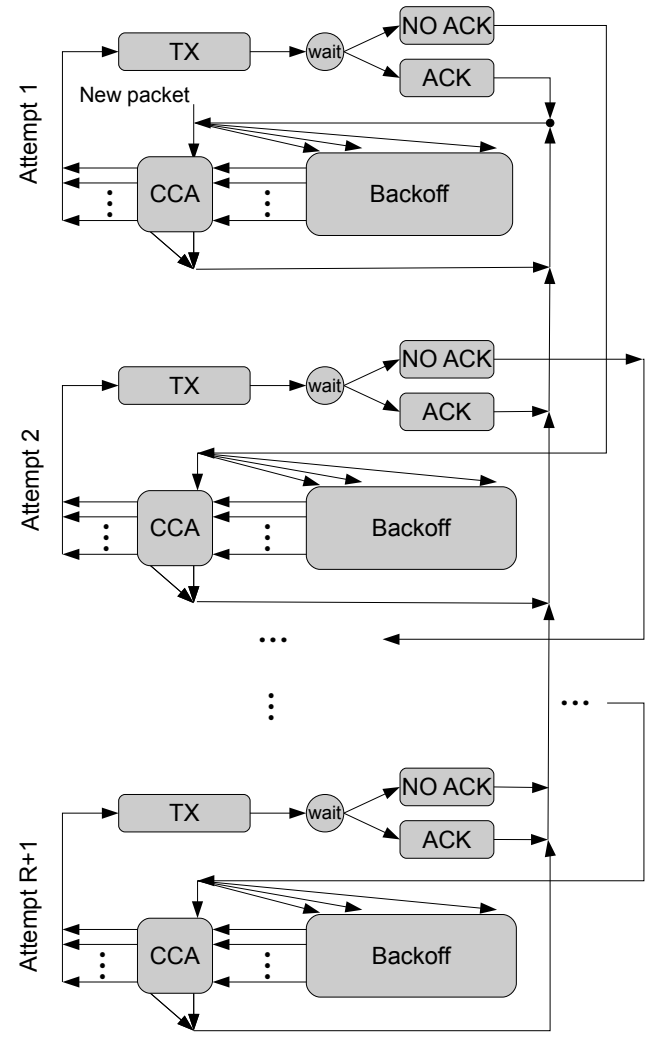

Fig. 2. Markov chain model for slotted CSMA/CA with retransmissions

rounded to a multiple of a slot, and since all operations have to start at the boundary of a slot, a node spends exactly one slot in waiting before the beginning of the reception of an ACK. A node always goes through this state, after a transmission, both in a successful and in a collided attempt. We assume that the transceiver is in idle mode during this slot. Otherwise, $c(t)$ represents the number of slots spent in waiting in vain for the ACK in a collided attempt when $c(t) \in\{-2,-1\}$, and in receiving the ACK in a successful attempt when $c(t) \in\{1,2\}$. As per the IEEE 802.15.4 standard, $L_{a c k}=2$ slots.

- When the node is in backoff, $c(t) \in\left\{0, \ldots, W_{i}-1\right\}$ represents the value of the backoff counter, where $W_{i}=$ $2^{\min \{m a c M i n B E+i, \operatorname{macMaxBE}\}}$ is the size of the backoff window at backoff stage $s(t)=i \in\{0, \ldots, M\}$.

- Finally, when the node is performing one of the CCAs, $c(t)$ represents the value of the CCA counter, with

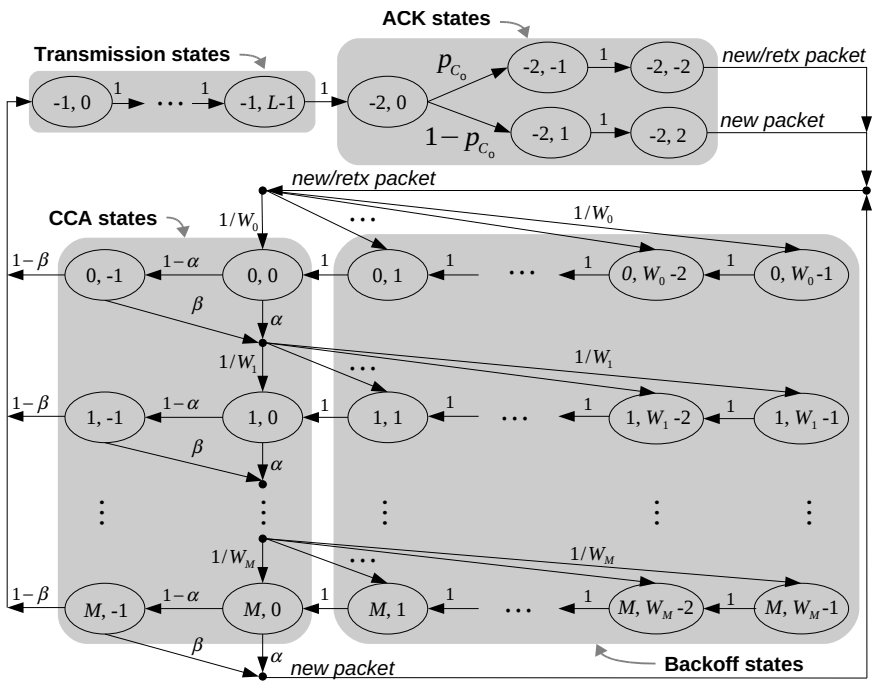

Fig. 3. Markov Model for slotted CSMA/CA per single transmission attempt.

$c(t)=0$ during $\mathrm{CCA}_{1}$ and $c(t)=-1$ during $\mathrm{CCA}_{2}$. Note that the state $\{s(t), c(t)\}=\{i, 0\}$, has to be seen as a $\mathrm{CCA}_{1}$ state and not as a backoff state as in the formulation in [7]. In fact, although the randomly picked backoff window size at stage $i$ can take any value in the set $\left\{0, \ldots, W_{i}-1\right\}$, the value zero indicates no waiting and immediate sensing.

Table I summarizes the values of $s(t)$ and $c(t)$ for the different states of the Markov chain.

The parameter $\alpha$ in Fig. 3 is the probability of assessing the channel busy during $\mathrm{CCA}_{1}$, and $\beta$ is the probability of assessing it busy during $\mathrm{CCA}_{2}$ given that it was idle in $\mathrm{CCA}_{1}$. As was mentioned earlier, in this per-node Markov chain model, the effect of other nodes on the behavior of a given node is captured only through the values of $\alpha$ and $\beta$ and, therefore, these two parameters play a key role. In all the Markov chain models available in the literature, it is assumed that the values of both $\alpha$ and $\beta$ are the same for different backoff stages. In reality, however, $\alpha$ and $\beta$ depend on the backoff stage in which the node is performing the sensing. We discuss this assumption in more detail in Section IV and refer to it as assumption A1.

\section{B. Calculating the Markov Chain Parameters}

Let $b_{i, k}$ be the steady-state probability of being in state $\{i, k\}$, i.e., $b_{i, k}=\lim _{t \rightarrow \infty} P\{s(t)=i, c(t)=k\}$. Вy inspecting Fig. 3, these steady-state probabilities can be seen 
to relate through the following equations:

$$
\begin{aligned}
& b_{i, k}=\frac{W_{i}-k}{W_{i}} b_{i, 0} \\
& 0 \leq i \leq M, 0 \leq k \leq W_{i}-1 \\
& b_{i, 0}=(1-y)^{i} b_{0,0} \\
& 1 \leq i \leq M \\
& b_{i,-1}=(1-\alpha) b_{i, 0} \\
& 0 \leq i \leq M \\
& b_{-1, k}=y \sum_{j=0}^{M} b_{j, 0}=y \phi \\
& 0 \leq k \leq L-1 \\
& b_{-2, k}=\left\{\begin{array}{l}
p_{\mathrm{c}_{\circ}} y \phi \\
y \phi \\
\left(1-p_{\mathrm{c}_{\circ}}\right) y \phi
\end{array}\right. \\
& \begin{array}{r}
-2 \leq k \leq-1 \\
k=0 \\
1 \leq k \leq 2
\end{array}
\end{aligned}
$$

where, for compactness, we have defined the access probability

$$
y=(1-\alpha)(1-\beta)
$$

and $\phi$ is the probability that a given node spends a randomly picked slot performing $\mathrm{CCA}_{1}$,

$$
\phi=\sum_{j=0}^{M} b_{j, 0}=\frac{1-(1-y)^{M+1}}{y} b_{0,0},
$$

and $p_{\mathrm{c}}$ (derived in Section $\mathrm{V}-\mathrm{C}$ ) is the probability that the transmission of a given node ends up in a collision, .

By taking into account the interactions between the $N$ nodes, expressions for $\alpha$ and $\beta$ were derived in [7]. In short, the probability $\alpha$ of finding the channel busy during $\mathrm{CCA}_{1}$ is

$$
\alpha=\left[L+L_{a c k}\left(1-p_{\mathrm{c}_{*}}\right)\right]\left[1-(1-\phi)^{N-1}\right] y
$$

where $p_{\mathrm{c}_{*}}$ (derived in Section $\mathrm{V}-\mathrm{C}$ ) is the probability that at a random slot a collision takes place in the network.

In turn, $\beta$, the probability that the channel is busy when the considered node does its second sensing, equals

$$
\begin{aligned}
\beta & =\left[1-\frac{2-p_{\mathrm{c}_{*}}}{2-p_{\mathrm{c}_{*}}+\frac{1}{1-(1-\phi)^{N}}}\right]\left(1-(1-\phi)^{N-1}\right) \\
& +\frac{1-p_{\mathrm{c}_{*}}}{2-p_{\mathrm{c}_{*}}+\frac{1}{1-(1-\phi)^{N}}} .
\end{aligned}
$$

The values of $\phi, \alpha$ and $\beta$ can be determined by imposing the normalizing condition

$$
\sum_{i} \sum_{k} b_{i, k}=1
$$

which, substituting $b_{i, k}$ from (1) and $b_{0,0}$ from (3), leads to a relationship that involves solely $\alpha, \beta$, and $\phi$. This relationship, together with (4), and (5), constitute a system of equations that is used in [7] to numerically obtain $\alpha, \beta$ and $\phi$. Instead, throughout this work, we obtain $\phi$ directly from simulation and use it to compute $\alpha$ and $\beta$ and validate the accuracy of our formulation for calculating different network metrics.

As mentioned earlier, in [7] the states $\{s(t), c(t)\}=\{i, 0\}$ are considered as backoff states, and an additional set of states are considered $\mathrm{CCA}_{1}$ states. Thus, in the normalizing condition (6), the states $b_{i, 0}$ - as per our notation-are counted twice in [7], which affects the values of $\alpha, \beta$, and $\phi$ obtained from the aforementioned system of equations.

As can be seen in Fig. 4, (4) and (5) offer a relatively good

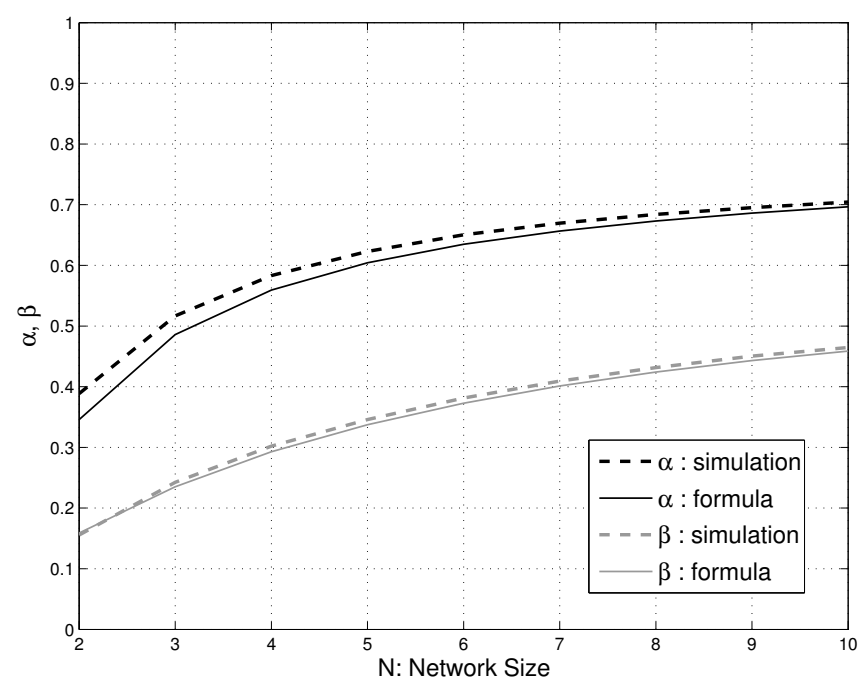

Fig. 4. Comparison between the values of $\alpha$ and $\beta$ obtained from (4) and (5), and those obtained from simulation.

approximation to the simulated values of $\alpha$ and $\beta$, specially for large network sizes.

\section{Events Notation}

Henceforth, we use the notation introduced in Table II to refer to events that can take place in the network. Except in the case of CF1 and CF2, the notation is in the general form of $\mathrm{C}_{X}$ where $\mathrm{C}$ stands for $\mathrm{CCA}_{1}$, and $X$ represents the number of nodes or the set of nodes involved. Replacing the $\mathrm{C}$ in this notation with TX indicates the corresponding nodes being in a transmission state, e.g., $\mathrm{TX}_{\supseteq i}$ is the event that exactly $i$ nodes are in transmission.

TABLE II

EVENTS NOTATION

\begin{tabular}{||c||l||}
\hline Notation & Event Description \\
\hline \hline $\mathrm{CF} 1$ & Channel Free in 1 slot \\
\hline $\mathrm{CF} 2$ & Channel Free in 2 consecutive slots \\
\hline $\mathrm{C}_{\supseteq\{i\}}$ & At least node $i$ in $\mathrm{CCA}_{1}$ \\
\hline $\mathrm{C}_{\geq i}$ & At least $i$ nodes in $\mathrm{CCA}_{1}$ \\
\hline $\mathrm{C}_{\{i\}}$ & Only node $i$ in $\mathrm{CCA}_{1}$ \\
\hline $\mathrm{C}_{i}$ & Exactly $i$ nodes in $\mathrm{CCA}_{1}$ \\
\hline$\widehat{\mathrm{C}}_{i}$ & Exactly $i$ nodes including node 1 in $\mathrm{CCA}_{1}$ \\
\hline
\end{tabular}

In the $\widehat{\mathrm{C}}_{i}$ notation and in general in the derivations hereafter, node 1 will be used as the reference node. This will be done only when the event under consideration is symmetric with respect to all nodes and, therefore, there is no loss of generality.

\section{Discussion OF ThE Key Assumptions}

In Section $\mathrm{V}$, we use the following simplifying assumptions in order to calculate the network metrics from the Markov chain model: 
A1. The probability to sense the channel busy during $\mathrm{CCA}_{1}$ and $\mathrm{CCA}_{2}$ does not depend on the backoff stage where the corresponding CCA is performed.

A2. The probability to sense the channel busy during $\mathrm{CCA}_{1}$ and $\mathrm{CCA}_{2}$ does not depend on $r$, the number of attempts the current packet has gone through. In other words, $\alpha$ and $\beta$ are constant for all transmission attempts of a given packet and, therefore, the Markov chains of different attempts are exactly the same.

A3. The probability of sensing the channel busy during a CCA does not depend on the random backoff value drawn in the backoff stage preceding the CCA;

A4. The probability $\phi$ that at a given time, a given node starts sensing the channel is independent of what other nodes in the network are doing at the same time;

A5. The probability of sensing the channel free in two consecutive slots equals $y=(1-\alpha)(1-\beta)$ from the per-node Markov chain. This assumption, which is expressed more precisely in Section IV-B, is fairly mild.

Assumptions A1-A3 are directly implied by the way we have constructed the Markov chain. A1 allows us to use the same $\alpha$ and $\beta$ value for all backoff stages of the chain. A2 basically implies that different transmission attempts on a packet are i.i.d, which is what enables us to simplify the Markov chain model from the one in Fig. 2 to the one in Fig. 3. Assumptions A4 and A5 simplify our calculations in Section V.

In all the Markov chain models of the IEEE 802.15.4 MAC proposed in the literature, wherever applicable, these assumptions are also made. In most cases, however, they are not explicitly stated as assumptions, but are rather considered as given facts. For example, in [7], A4 is the only stated assumption, even though the rest of the assumptions (except for A2, which does not apply) are also made. Furthermore, in all the works where retransmissions are modeled ( [6], [8], [9], and [11]) it is always assumed that different attempts on a packet are i.i.d, as in A2.

These assumptions greatly simplify the model and the derivations, and often lead to relatively good results when compared to simulation. However, as we will see in Section VI, for certain network metrics (e.g., packet-discard probability and throughput) the values of the metrics calculated using these assumptions have noticeable differences compared to their values from simulation, specially for small networks. This is despite the fact that $\alpha$ and $\beta$ match well their value from simulation, as we saw in Fig. 4. To explain this behavior, in the remainder of this section, we introduce some new parameters that will only be used in Section VI and later.

\section{A. Sensing probabilities for different backoff stages: $\alpha_{i}, \beta_{i}$}

Let $\alpha_{i}$ and $\beta_{i}$ denote the probabilities of sensing the channel busy during $\mathrm{CCA}_{1}$ and $\mathrm{CCA}_{2}$, respectively, for each backoff stage $i$. As was mentioned earlier, in the Markov chain model of Fig. 3, it was assumed that $\alpha_{i}=\alpha$ and $\beta_{i}=\beta$ for all $i \in\{0 \cdots M\}$ (A1). This greatly simplifies the analysis. However, as seen in Fig. 5, $\alpha$ and $\beta$ are in fact very dependent

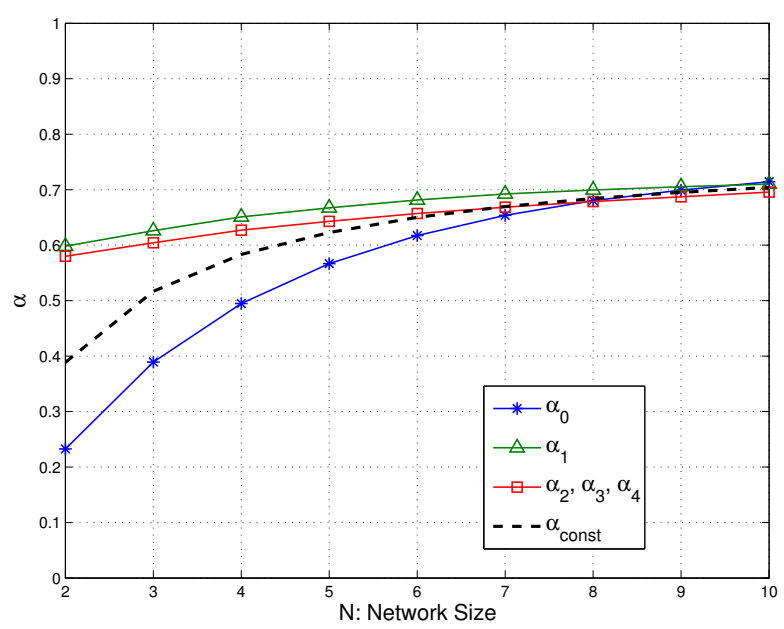

(a) $\alpha$ for different backoff stages

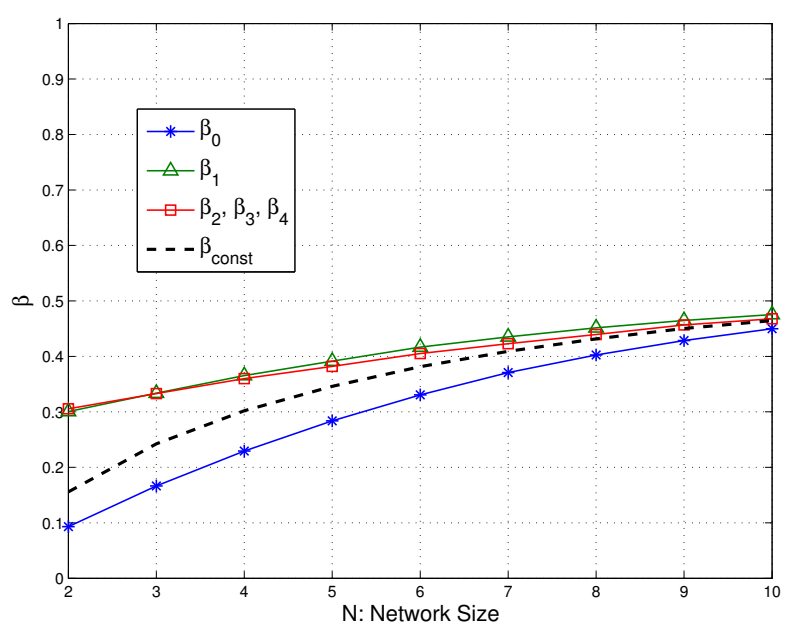

(b) $\beta$ for different backoff stages

Fig. 5. Dependence of $\alpha$ and $\beta$ on the backoff stage.

on the backoff stage. This difference is particularly noticeable between the first backoff stage $(i=0)$ and the rest of the backoff stages. This can be explained by observing that a node in the first backoff stage on average draws a smaller backoff value than other nodes that are competing with it. Additionally, as explained in [13], even though the probability of a node being in the first backoff stage is not small (a node goes through the first backoff stage once per every attempt), the joint probability of two nodes being in the first backoff stage is very small. This means that a node in the first backoff stage is given better opportunities than nodes in any other stage. Thus, it is very likely for it to find the channel idle. This explains why $\alpha_{0}$ and $\beta_{0}$ are different from $\alpha_{i}$ and $\beta_{i}$ for $i>0$.

Referring back to the $\alpha$ and $\beta$ derived in Section III-B, they can be viewed as unconditional versions of $\alpha_{i}$ and $\beta_{i}$, which are conditioned on the backoff stage. As function of $\alpha_{i}$ and 
$\beta_{i}, \alpha$ can be expressed

$$
\begin{aligned}
1-\alpha & =\operatorname{Pr}\left\{\mathrm{CF} 1 \mid \mathrm{C}_{\supseteq\{1\}}\right\} \\
& =\operatorname{Pr}\left\{\mathrm{CF} 1 \mid \bigcup_{i=0}^{M}\{\text { Node } 1 \text { in state }(i, 0)\}\right\} \\
& =\frac{\sum_{i=0}^{M} \operatorname{Pr}\{\mathrm{CF} 1 \mid \text { Node } 1 \text { in state }(i, 0)\} b_{i, 0}}{\sum_{i=0}^{M} b_{i, 0}} \\
& =\frac{\sum_{i=0}^{M}\left(1-\alpha_{i}\right) b_{i, 0}}{\phi}
\end{aligned}
$$

while, in turn, $\beta$ can be expressed as

$$
\begin{aligned}
1-\beta & =\operatorname{Pr}\left\{\mathrm{CF} 1 \mid \text { Node } 1 \text { in } \mathrm{CCA}_{2}\right\} \\
& =\operatorname{Pr}\left\{\mathrm{CF} 1 \mid \bigcup_{i=0}^{M}\{\text { Node } 1 \text { in state }(i,-1)\}\right\} \\
& =\frac{\sum_{i=0}^{M} \operatorname{Pr}\{\mathrm{CF} 1 \mid \text { Node } 1 \text { in state }(i,-1)\} b_{i,-1}}{\sum_{i=0}^{M} b_{i,-1}} \\
& =\frac{\sum_{i=0}^{M}\left(1-\beta_{i}\right)\left(1-\alpha_{i}\right) b_{i, 0}}{(1-\alpha) \phi}
\end{aligned}
$$

From (8) we can directly obtain the following equality for $y=(1-\alpha)(1-\beta)$ :

$$
y=\frac{1}{\phi} \sum_{i=0}^{M}\left(1-\beta_{i}\right)\left(1-\alpha_{i}\right) b_{i, 0}
$$

This means that, in Sections V and VI, whenever the metric being calculated does not directly depend on the particular backoff stage where the CCAs are performed, using $\alpha$ and $\beta$ will not amount to any inaccuracy. This is due to the fact that, in these cases, $\alpha_{i}$ and $\beta_{i}$ only appear through the form $\sum_{i=0}^{M}\left(1-\beta_{i}\right)\left(1-\alpha_{i}\right) b_{i, 0}$, which, as per (9), can be directly replaced with $y \phi$ without invoking A1. This is the main reason why, as we will see, the only network metrics affected by A1 are average delay and average power consumption, where we need to calculate the expected time spent in backoff and CCA.

\section{B. Refining the access probability: $y_{\circ}$ and $y_{*}$}

Let $y_{\circ}$ be the probability that a given node in $\mathrm{CCA}_{1}$ senses the channel free for two consecutive slots. In other words,

$$
y_{\circ}=\operatorname{Pr}\left\{\mathrm{CF} 2 \mid \mathrm{C}_{\supseteq\{1\}}\right\} .
$$

This probability is conditioned on the fact that at least node 1 is in $\mathrm{CCA}_{1}$, and therefore other nodes could be in any state, including $\mathrm{CCA}_{1}$.

Note that $y_{\circ}$ is equivalent to $y=(1-\alpha)(1-\beta)$ as defined in Section III, i.e., the probability that when a node reaches a $\mathrm{CCA}_{1}$ state in its Markov chain, it finds the channel free for two consecutive slots. Since this probability is only conditioned on a given node being in $\mathrm{CCA}_{1}$, and there is no conditioning on what the rest of the nodes are doing, by definition we have $y_{\circ}=y$.

We then define $y_{*}$ as

$$
y_{*}=\operatorname{Pr}\left\{\mathrm{CF} 2 \mid \mathrm{C}_{\geq 1}\right\}
$$

which is the probability that the channel is sensed free for two consecutive slots, given that at least one node was in $\mathrm{CCA}_{1}$. These probabilities, $y_{\circ}$ and $y_{*}$, have a subtle but important difference. To see this, we define

$$
y_{(i)}=\operatorname{Pr}\left\{\mathrm{CF} 2 \mid \mathrm{C}_{i}\right\}
$$

which is the probability that the channel is free for two slots, given that exactly $i$ nodes are in $\mathrm{CCA}_{1}$. Then $y_{\circ}$ can be calculated as

$$
\begin{aligned}
y_{\circ} & =\operatorname{Pr}\left\{\mathrm{CF} 2 \mid \mathrm{C}_{\supseteq\{1\}}\right\}=\operatorname{Pr}\left\{\mathrm{CF} 2 \mid \bigcup_{i=1}^{N} \widehat{\mathrm{C}}_{i}\right\} \\
& =\frac{\sum_{i=1}^{N} \operatorname{Pr}\left\{\widehat{\mathrm{C}}_{i}\right\} \operatorname{Pr}\left\{\mathrm{CF} 2 \mid \widehat{\mathrm{C}}_{i}\right\}}{\sum_{i=1}^{N} \operatorname{Pr}\left\{\widehat{\mathrm{C}}_{i}\right\}} \\
& =\frac{\sum_{i=1}^{N}\left(\begin{array}{c}
N-1 \\
i-1
\end{array}\right) \phi^{i}(1-\phi)^{N-i} y_{(i)}}{\sum_{i=1}^{N}\left(\begin{array}{c}
N-1 \\
i-1
\end{array}\right) \phi^{i}(1-\phi)^{N-i}} .
\end{aligned}
$$

where (13) is due to disjointness of $\widehat{\mathrm{C}}_{i}$, and (14) is reached by replacing $\operatorname{Pr}\left\{\mathrm{CF} 2 \mid \widehat{\mathrm{C}}_{i}\right\}$ with $y_{(i)}$. This can be done because $\operatorname{Pr}\left\{\mathrm{CF} 2 \mid \widehat{\mathrm{C}}_{i}\right\}=\operatorname{Pr}\left\{\mathrm{CF} 2 \mid \mathrm{C}_{i}\right\}=y_{(i)}$. To see this, note that $\mathrm{C}_{i}$ can be written as the union of events in the form of $\mathrm{C}_{\left\{j, k_{1} \cdots k_{i-1}\right\}}$ with $j=1 \cdots N$ and $k_{j}$ ordered such that $j<k_{1}<\cdots<k_{i-1}$. These events are symmetric and disjoint with respect to the event CF2. Furthermore, $\widehat{\mathrm{C}}_{i}$ can also be written as the union of the subset of the same events with $j=1$. Therefore, from the Lemma 1 in Appendix A, $\operatorname{Pr}\left\{\mathrm{CF} 2 \mid \widehat{\mathbf{C}}_{i}\right\}=\operatorname{Pr}\left\{\mathrm{CF} 2 \mid \mathrm{C}_{\left\{1, k_{1} \cdots k_{i-1}\right\}}\right\}=\operatorname{Pr}\left\{\mathrm{CF} 2 \mid \mathrm{C}_{i}\right\}$.

In turn, $y_{*}$ is given by

$$
\begin{aligned}
y_{*} & =\operatorname{Pr}\left\{\mathrm{CF} 2 \mid \mathrm{C}_{\geq 1}\right\}=\operatorname{Pr}\left\{\mathrm{CF} 2 \mid \bigcup_{i=1}^{N} \mathrm{C}_{i}\right\} \\
& =\frac{\sum_{i=1}^{N} \operatorname{Pr}\left\{\mathrm{C}_{i}\right\} \operatorname{Pr}\left\{\mathrm{CF} 2 \mid \mathrm{C}_{i}\right\}}{\sum_{i=1}^{N} \operatorname{Pr}\left\{\mathrm{C}_{i}\right\}} \\
& =\frac{\sum_{i=1}^{N}\left(\begin{array}{c}
N \\
i
\end{array}\right) \phi^{i}(1-\phi)^{N-i} y_{(i)}}{\sum_{i=1}^{N}\left(\begin{array}{c}
N \\
i
\end{array}\right) \phi^{i}(1-\phi)^{N-i}} .
\end{aligned}
$$

where (15) is due to disjointness of $\mathrm{C}_{i}$.

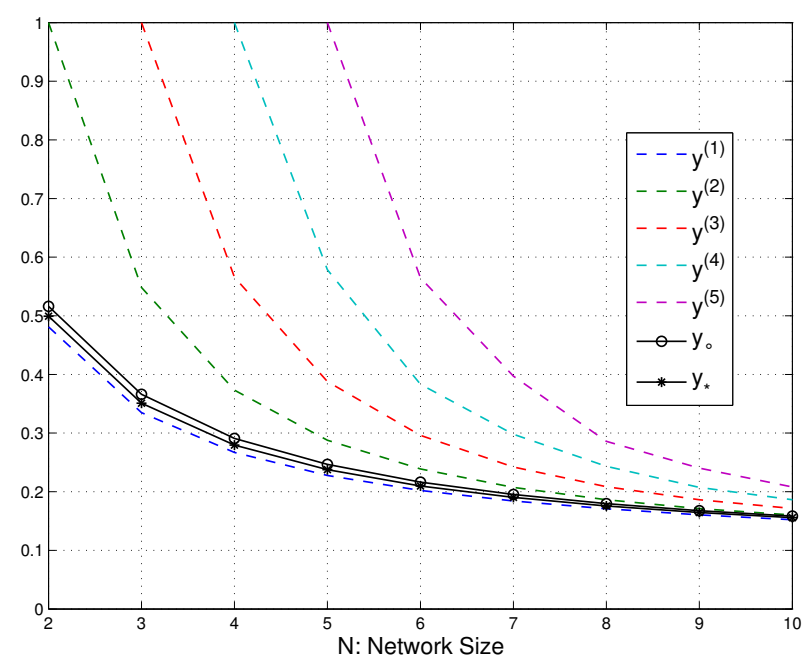

Fig. 6. Comparison between different $y$ values obtained from simulation. 
Fig. 6 contrasts the different $y$ values introduced above, as obtained from simulation.

We can now use this notation to express A5 more precisely as stating that $y_{\circ}=y_{*}=y_{(1)}=y$. All these quantities are close (cf. Fig. 6), and that motivates A5, which is used in Section $\mathrm{V}$ and in all the works reported from the literature. It will be shown in Section VI that, indeed, A5 has negligible impact on a number of network metrics, but it has a significant effect on other metrics.

\section{Calculating Network Metrics Using the Traditional Markov Chain Assumptions}

In this section, we use the Markov chain model and Assumptions A1-A5 to calculate different network metrics as a function of $\phi$ and $y$ (or, equivalently, $\phi, \alpha$ and $\beta$ ).

\section{A. Throughput}

We define the per-node throughput, $S_{\circ}$, as the fraction of time that a node spends in successful transmission. ${ }^{4}$ Alternatively, the network throughput, $S_{*}$, is defined, as in [7], as the fraction of time that the network spends in successful transmission, i.e., the fraction of time in which exactly one node is transmitting:

$$
\begin{aligned}
S_{*} & =N S_{\circ} \\
& =\operatorname{Pr}\left\{\text { at } t: \operatorname{TX}_{1}\right\} \\
& =N \operatorname{Pr}\left\{\text { at } t: \operatorname{TX}_{\{1\}}\right\} \\
& =N L \operatorname{Pr}\left\{\text { at } t^{\prime} \in[t-1-L, t-2]: \mathrm{C}_{\{1\}} \cap \mathrm{CF} 2\right\}
\end{aligned}
$$

where (17) and (18) are due to symmetry, and (19) holds because, if node 1 is transmitting at time $t$, it must have been sensing the channel at some time $t^{\prime}$ prior to $t$, and the channel must have been free for two consecutive slots $\left(t^{\prime}\right.$ and $\left.t^{\prime}+1\right){ }^{5}$ Using A5, (19) simplifies to [7]

$$
S_{*}=N L \phi(1-\phi)^{N-1} y .
$$

Note that $S_{*}$ can equivalently be viewed as the network success probability, i.e., the probability that at a random time, a successful transmission is taking place in the network.

\section{B. Transmission Probability}

Let $p_{\mathrm{tx}}$ 。 denote the probability that, at a random slot, a particular node is transmitting. This probability is the same for all nodes due to symmetry and can be written as

$$
\begin{aligned}
p_{\mathrm{tx}_{\circ}} & =\operatorname{Pr}\left\{\mathrm{TX}_{\supseteq\{1\}}\right\} \\
& =L \operatorname{Pr}\left\{\mathrm{C}_{\supseteq\{1\}} \cap \mathrm{CF} 2\right\} .
\end{aligned}
$$

Using A5,

$$
p_{\mathrm{tx}_{\circ}}=L \phi y .
$$

Note that, given $p_{\mathrm{tx}_{\circ}}$, one cannot directly obtain $p_{\mathrm{tx}_{*}}$, the probability that one or more nodes in the network are

\footnotetext{
${ }^{4}$ To convert fraction of time into bits/s, the throughput must be multiplied by $25 \times 10^{3}$, which is the number of bits per slot divided by the slot duration.

${ }^{5}$ Since the time can usually be deduced from the context, it is henceforth dropped from the notation.
}

transmitting at a random slot. This is because $p_{\mathrm{t} \mathrm{x}_{\circ}}$ does not provide any information on the number of other nodes that are transmitting at the same time as the considered one. The network transmission probability, $p_{\mathrm{tx}_{*}}$, can be calculated as

$$
\begin{aligned}
p_{\mathrm{tx}_{*}} & =\operatorname{Pr}\left\{\mathrm{TX}_{\geq 1}\right\} \\
& =L \operatorname{Pr}\left\{\mathrm{C}_{\geq 1} \cap \mathrm{CF} 2\right\} .
\end{aligned}
$$

Invoking A5 again [7]

$$
p_{\mathrm{tx}_{*}}=L\left[1-(1-\phi)^{N}\right] y .
$$

\section{Collision Probability}

The per-node collision probability, $p_{\mathrm{c}_{\circ}}$, is defined as the probability that at a time in which a given node, e.g., node 1 , is transmitting, one or more other nodes are also transmitting. This can happen only if, when node 1 was performing the $\mathrm{CCA}_{1}$ that led to the current transmission, another node was also in $\mathrm{CCA}_{1}$. In other words,

$$
\begin{aligned}
p_{\mathrm{c}_{\circ}} & =\operatorname{Pr}\left\{\mathrm{TX}_{\geq 2} \mid \mathrm{TX}_{\supseteq\{1\}}\right\} \\
& =\operatorname{Pr}\left\{\mathrm{C}_{\geq 2} \mid \mathrm{C}_{\supseteq\{1\}} \cap \mathrm{CF} 2\right\}
\end{aligned}
$$

Using A4, this simplifies to

$$
\begin{aligned}
p_{\mathrm{c}} & =\operatorname{Pr}\{\text { at least one of } N-1 \text { nodes sensing }\} \\
& =1-(1-\phi)^{N-1}
\end{aligned}
$$

The network collision probability, $p_{\mathrm{c}_{*}}$, is the probability that at a time in which at least one node is transmitting, one or more other nodes are also transmitting:

$$
\begin{aligned}
p_{\mathrm{c}_{*}} & =\operatorname{Pr}\left\{\mathrm{TX}_{\geq 2} \mid \mathrm{TX}_{\geq 1}\right\} \\
& =1-\operatorname{Pr}\left\{\mathrm{TX}_{1} \mid \mathrm{TX}_{\geq 1}\right\} \\
& =1-\frac{S_{*}}{p_{\mathrm{tx}_{*}}} .
\end{aligned}
$$

Replacing $S_{*}$ from (20) and $p_{\mathrm{tx}_{*}}$ from (24), we obtain the same expression as in [7], i.e.,

$$
p_{\mathbf{c}_{*}}=1-\frac{N \phi(1-\phi)^{N-1}}{1-(1-\phi)^{N-1}} .
$$

Since the time intervals that different nodes spend in collision overlap, and the amount of overlap is not captured by $p_{\mathrm{c}_{\circ}}$, the relationship between $p_{\mathrm{c}_{\circ}}$ and $p_{\mathrm{c}_{*}}$ is not a trivial one. This can be seen comparing (26) and (28).

\section{Packet Discard Probability}

The packet discard probability, $p_{d}$, is the fraction of generated packets that are not transmitted with success.

To compute $p_{d}$, let us first focus on what happens on an individual transmission attempt. An attempt can end either in channel access failure, in collision, or in successful transmission (cf. Sections II-III), to which hereafter we refer as FAIL, COL, and SUC, respectively. The probabilities that an attempt ends in FAIL, COL and SUC are respectively

$$
\begin{aligned}
& p_{\text {FAIL }}=(1-y)^{M+1} \\
& p_{\text {COL }}=p_{\mathrm{c}_{\circ}}\left(1-p_{\text {FAIL }}\right) \\
& p_{\text {SUC }}=\left(1-p_{\mathrm{c}_{\circ}}\right)\left(1-p_{\text {FALL }}\right) .
\end{aligned}
$$


A packet may require $i$ transmission attempts, with $1 \leq i \leq$ $R+1$, to be successfully transmitted. A packet is discarded if all the $R+1$ possible attempts end in COL or if one attempt ends in FAIL. Thus,

$$
p_{d}=p_{d c}+p_{d f}
$$

where $p_{d c}$ and $p_{d f}$ are the probabilities that a packet is discarded due to reaching the retransmission limit $R$ or due to FAIL, respectively. These probabilities are

$$
\begin{aligned}
p_{d c} & =\operatorname{Pr}\left\{A_{1}, \cdots, A_{R+1}: \mathrm{COL}\right\} \\
& =p_{\mathrm{COL}}^{R+1}
\end{aligned}
$$

and

$$
\begin{aligned}
p_{d f} & =\sum_{i=0}^{R} \operatorname{Pr}\left\{A_{1}, \cdots, A_{i}: \mathrm{COL} \cap A_{i+1}: \text { FAIL }\right\} \\
& =\sum_{i=0}^{R} p_{\mathrm{FALL}} p_{\mathrm{COL}}^{i} \\
& =p_{\mathrm{FAIL}} \frac{1-p_{\mathrm{COL}}^{R+1}}{1-p_{\mathrm{COL}}}
\end{aligned}
$$

where $A_{i}$ indicates the $i^{\text {th }}$ attempt. Equalities (34) and (36) are due to $\mathrm{A} 2$.

Finally, the packet discard probability is given by

$$
p_{d}=p_{\mathrm{COL}}^{R+1}+p_{\mathrm{FAIL}} \frac{1-p_{\mathrm{COL}}^{R+1}}{1-p_{\mathrm{COL}}}
$$

It should be mentioned that $p_{d}$ is also calculated in [9]. However, to calculate $p_{d f}$, [9] only takes into account the potential failure at the first attempt. Furthermore, when calculating $p_{d c}$, [9] uses the probability that a given slot is spent in collision in place of the probability that an attempt collides. These result in an incorrect formulation for $p_{d}=p_{d c}+p_{d f}$. In [7], the packet discard probability $p_{d}$ is not computed, but the failure probability is given as $p_{\text {FAIL }}=b_{0,0}(1-y)^{M+1}$. Since an attempt always has to go through the state $\{0,0\}$, the extra term $b_{0,0}$ results in an incorrect formulation for the probability of an attempt ending in failure.

\section{E. Power Consumption}

At a given instant in time, depending on whether a node is idle, receiving, or transmitting, it uses different power levels denoted by $P_{i d}, P_{r x}$, and $P_{t x}$, respectively. Thus, the average power consumption per node is given by

$$
\bar{P}=\frac{\bar{n}_{B} P_{i d}+\bar{n}_{C} P_{r x}+\left(1-p_{\mathrm{FAIL}}\right)\left[\left(P_{i d}+2 P_{r x}\right)+L P_{t x}\right]}{\bar{n}_{B}+\bar{n}_{C}+(3+L)\left(1-p_{\mathrm{FAIL}}\right)}
$$

where $\bar{n}_{B}$ and $\bar{n}_{C}$ are the average number of slots spent per attempt in backoff and CCA, respectively, and are derived in Appendix B. The transceiver is idle when the node is either in backoff or in the first ACK state. It is in reception mode when the node is either performing CCA, or waiting for or receiving an ACK, and it is in transmission mode only when the node is transmitting a packet. The last term in the numerator accounts for the energy spent in transmission and ACK reception, and, therefore, it is multiplied by $\left(1-p_{\text {FAIL }}\right)$, the probability that an attempt ends in transmission (and not a failure). Finally, the denominator is the average number of slots spent per attempt.

\section{F. Delay}

The average delay for a successfully transmitted packet, i.e. the number of slots it takes from the moment it reaches the head of the line to the moment it arrives at its destination, is

$$
\bar{D}=\left(\bar{n}_{B_{t x}}+\bar{n}_{C_{t x}}+L+3\right)\left(\bar{r}_{s u c}+1\right)-3
$$

where $\bar{n}_{B_{t x}}$ and $\bar{n}_{C_{t x}}$ are the mean number of slots spent performing backoff and CCA, respectively, during an attempt that ends in transmission. They are derived in Appendix B. $\bar{r}_{\text {suc }}$ is the average number of times a packet has to be retransmitted until it is transmitted with success. For each transmission attempt that ends in either COL or SUC, a node spends $L$ slots transmitting the packet and 3 slots waiting for the ACK.

A packet has $R+1$ possible transmission attempts, and therefore it can be transmitted with success after colliding in up to $R$ attempts. Therefore, $\bar{r}_{\text {suc }}$ can be computed as

$$
\begin{aligned}
& \bar{r}_{\text {suc }}= \sum_{i=0}^{R} i \operatorname{Pr}\left\{A_{1} \cdots A_{i}: \operatorname{COL} \cap A_{i+1}: \mathrm{SUC} \mid \mathrm{pkt} \mathrm{SUC}\right\} \\
&=\sum_{i=0}^{R} i \operatorname{Pr}\left\{\mathrm{pkt} \mathrm{SUC} \mid A_{1} \cdots A_{i}: \mathrm{COL} \cap A_{i+1}: \mathrm{SUC}\right\} \\
& \times \frac{\operatorname{Pr}\left\{A_{1} \cdots A_{i}: \mathrm{COL} \cap A_{i+1}: \mathrm{SUC}\right\}}{\operatorname{Pr}\{\mathrm{pkt} \mathrm{SUC}\}}
\end{aligned}
$$

Since, if a packet is not discarded, it must have been transmitted successfully, the probability that a packet is successfully transmitted is

$$
\operatorname{Pr}\{\text { pkt SUC }\}=1-p_{d}=p_{\text {SUC }} \frac{1-p_{\mathrm{COL}}^{R+1}}{1-p_{\mathrm{COL}}} .
$$

Finally, based on A2,

$$
\begin{aligned}
\bar{r}_{\text {suc }} & =\sum_{i=0}^{R} i \frac{p_{\mathrm{SUC}} p_{\mathrm{COL}}^{i}}{1-p_{d}} \\
& =\frac{p_{\mathrm{SUC}}}{1-p_{d}} \cdot p_{\mathrm{COL}} \sum_{i=0}^{R} i p_{\mathrm{COL}}^{i-1} \\
& =\frac{1-p_{\mathrm{COL}}}{1-p_{\mathrm{COL}}^{R+1}} \cdot p_{\mathrm{COL}} \cdot \frac{1-(R+1) p_{\mathrm{COL}}^{R}+R p_{\mathrm{COL}}^{R+1}}{\left(1-p_{\mathrm{COL}}\right)^{2}} \\
& =p_{\mathrm{COL}} \cdot \frac{1-(R+1) p_{\mathrm{COL}}^{R}+R p_{\mathrm{COL}}^{R+1}}{\left(1-p_{\mathrm{COL}}^{R+1}\right)\left(1-p_{\mathrm{COL}}\right)} .
\end{aligned}
$$

\section{BEyOnd THE TRADITIONAL MARKOV CHAIN ASSUMPTIONS}

In the previous section, we derived the network metrics using A1-A5 in Section IV. These assumptions enabled calculating $\alpha$ and $\beta$ as a function of $\phi$. This is because $p_{\mathrm{c}_{*}}$, calculated in this fashion as per (28), is only a function of $\phi$, which in turn means that $\beta$ is also only a function of $\phi$, from which $\alpha$ can be directly calculated. These parameters can then be used to calculate all the relevant network metrics. In this section we see, through comparison with simulation results (cf. 
Appendix D), that, although the formulation obtained using these assumptions gives a relatively good approximation for certain metrics (e.g., power consumption and delay), there is a larger discrepancy between analytical and simulation results for some other metrics (e.g., transmission, collision, packet discard probabilities, and throughput).

In what follows, we look at the metrics derived in the previous section and single out the assumptions that have the most impact on each one. We then calculate the same metrics, without those assumptions, and see that the new formulation offers more accurate results. However, in this case, only a semi-analytical approach is possible since, in addition to $\phi$, one needs to obtain the parameters defined in Sections IV-A and IV-B also from simulation as no explicit formulation is derived for them.

\section{A. Throughput}

The network throughput, from (19), is given by

$$
\begin{aligned}
S_{*} & =N L \operatorname{Pr}\left\{\mathrm{C}_{\{1\}} \cap \mathrm{CF} 2\right\} \\
& =N L \operatorname{Pr}\left\{\mathrm{C}_{\{1\}}\right\} \operatorname{Pr}\left\{\mathrm{CF} 2 \mid \mathrm{C}_{\{1\}}\right\} \\
& =N L \phi(1-\phi)^{N-1} y_{(1)} .
\end{aligned}
$$

As before, $S_{*}=N S_{\circ}$. Comparing (20) and (43), we see that the difference between simulation and formula (cf. Fig. 7) is only due to the use of different $y$ values. Even though the difference between $y_{\circ}$ and $y_{(1)}$ is relatively small, we see here that it gives rise to a discrepancy of more than $10 \%$ in the network throughput for small networks. Using the appropriate $y$, i.e., $y_{(1)}$, the discrepancy between simulation and formula almost completely vanishes.

Notice that the per-node throughput drops rapidly with the network size, which justifies focusing on relatively small networks.

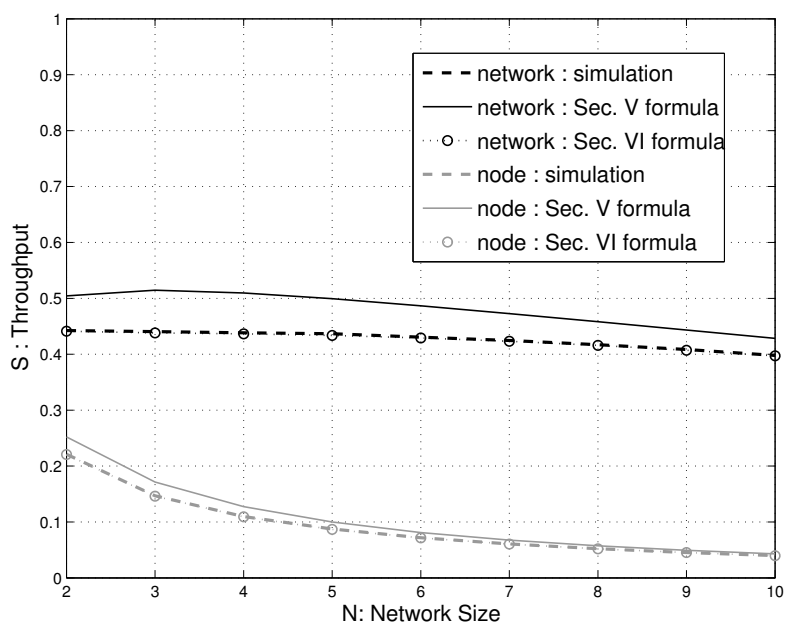

Fig. 7. Comparison between simulation and formula for $S_{*}$ and $S_{\circ}$.

\section{B. Transmission Probability}

From (21),

$$
\begin{aligned}
p_{\mathrm{tx}_{\circ}} & =L \operatorname{Pr}\left\{\mathrm{C}_{\supseteq\{1\}} \cap \mathrm{CF} 2\right\} \\
& =L \operatorname{Pr}\left\{\mathrm{C}_{\supseteq\{1\}}\right\} \operatorname{Pr}\left\{\mathrm{CF} 2 \mid \mathrm{C}_{\supseteq\{1\}}\right\} \\
& =L \phi y_{\circ}=L \phi y
\end{aligned}
$$

which means that the per-node transmission probability in (22) was already characterized without the need for any of the assumptions. This can also be seen in Fig. 8, where the small difference between simulation and formula is due to the approximations in calculating $\alpha$ and $\beta$ which directly affect $y_{\circ}=(1-\alpha)(1-\beta)$. (23)

However, for the network transmission probability, from

$$
\begin{aligned}
p_{\mathrm{tx}_{*}} & =L \operatorname{Pr}\left\{\mathrm{C}_{\geq 1} \cap \mathrm{CF} 2\right\} \\
& =L \operatorname{Pr}\left\{\mathrm{C}_{\geq 1}\right\} \operatorname{Pr}\left\{\mathrm{CF} 2 \mid \mathrm{C}_{\geq 1}\right\} \\
& =L\left[1-(1-\phi)^{N}\right] y_{*} .
\end{aligned}
$$

As evidenced in Fig. 8, using $y_{\circ}$ instead of $y_{*}$ in this case results in a relatively large error in $p_{\mathrm{tx}_{*}}$.

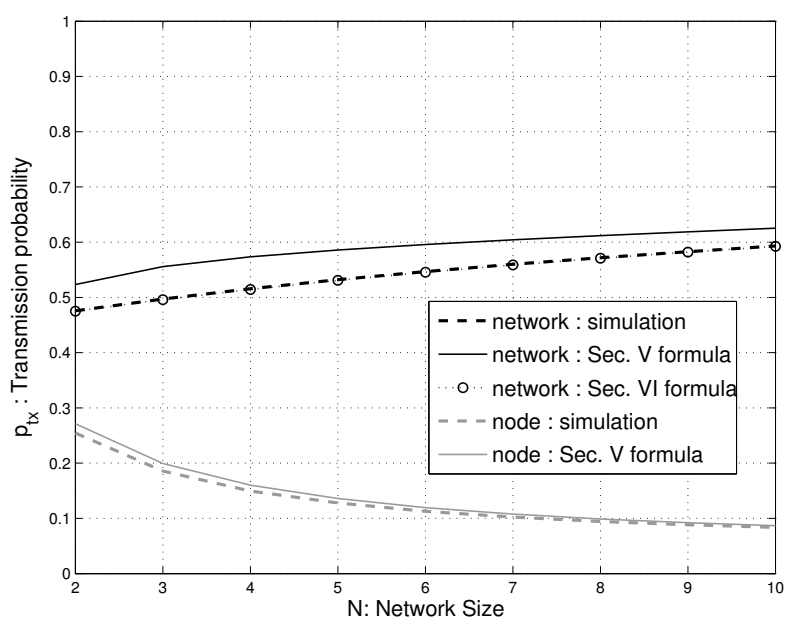

Fig. 8. Comparison between simulation and formula for $p_{\mathrm{tx}_{\circ}}$ and $p_{\mathrm{tx}_{*}}$.

\section{Collision Probability}

As can be seen in Fig. 9, one of the metrics most affected by A1-A5 is the collision probability, both per-node and pernetwork, and especially for small networks.

The per node collision probability, without using A1-A5, can be calculated from (25) as

$$
\begin{aligned}
& p_{\mathrm{c}_{\circ}}=\operatorname{Pr}\left\{\mathrm{TX}_{\geq 2} \mid \mathrm{TX}_{\supseteq\{1\}}\right\}=\operatorname{Pr}\left\{\mathrm{C}_{\geq 2} \mid \mathrm{C}_{\supseteq\{1\}} \cap \mathrm{CF} 2\right\} \\
& =\frac{\operatorname{Pr}\left\{\mathrm{C}_{\geq 2} \cap \mathrm{C}_{\supseteq\{1\}} \cap \mathrm{CF} 2\right\}}{\operatorname{Pr}\left\{\mathrm{C}_{\supseteq\{1\}} \cap \mathrm{CF} 2\right\}}=\frac{\operatorname{Pr}\left\{\mathrm{C}_{\geq 2} \cap \mathrm{C}_{\supseteq\{1\}} \cap \mathrm{CF} 2\right\}}{p_{\mathrm{tx}_{\circ}} / L} \\
& =\frac{\operatorname{Pr}\left\{\mathrm{CF} 2 \cap\left[\cup_{i=2}^{N}\left(\mathrm{C}_{\supseteq\{1\}} \cap \mathrm{C}_{i}\right)\right]\right\}}{\phi y_{\circ}} \\
& =\frac{\operatorname{Pr}\left\{\mathrm{CF} 2 \cap\left[\cup_{i=2}^{N} \widehat{\mathrm{C}}_{i}\right]\right\}}{\phi y_{\circ}} .
\end{aligned}
$$


Since the events $\widehat{\mathrm{C}}_{i}=\mathrm{C}_{\supseteq\{1\}} \cap \mathrm{C}_{i}$ are disjoint for different values of $i$,

$$
\begin{aligned}
p_{\mathrm{c}_{\circ}} & =\frac{\sum_{i=2}^{N} \operatorname{Pr}\left\{\mathrm{CF} 2 \mid \widehat{\mathrm{C}}_{i}\right\} \operatorname{Pr}\left\{\widehat{\mathrm{C}}_{i}\right\}}{\phi y_{\circ}} \\
& =\frac{\sum_{i=2}^{N} y_{(i)}\left(\begin{array}{c}
N-1 \\
i-1
\end{array}\right) \phi^{i}(1-\phi)^{N-i}}{\phi y_{\circ}} \\
& =\frac{\phi\left[y_{\circ}-y_{(1)}(1-\phi)^{N-1}\right]}{\phi y_{\circ}} \\
& =1-\frac{y_{(1)}}{y_{\circ}}(1-\phi)^{N-1}
\end{aligned}
$$

The network collision probability, from (27), is given by

$$
\begin{aligned}
p_{\mathrm{c}_{*}} & =1-\frac{S_{*}}{p_{\mathrm{tx}_{*}}}=1-\frac{N L \phi(1-\phi)^{N-1} y_{(1)}}{L\left[1-(1-\phi)^{N}\right] y_{*}} \\
& =1-\frac{N \phi(1-\phi)^{N-1} y_{(1)}}{\left[1-(1-\phi)^{N}\right] y_{*}} .
\end{aligned}
$$

Note that, using A5, $y_{(1)}=y_{*}=y$ and therefore $y_{(1)}$ and $y_{*}$ would cancel out in (49). In Section V-C, this enabled calculating $p_{\mathrm{c}_{*}}$ without needing $\alpha$ and $\beta$, and subsequently calculating $\alpha$ and $\beta$ in the ACK mode. However, this is no longer possible without A5 because, to calculate $p_{\mathrm{c}_{*}}$, we need $\alpha$ and $\beta$, and vice versa.

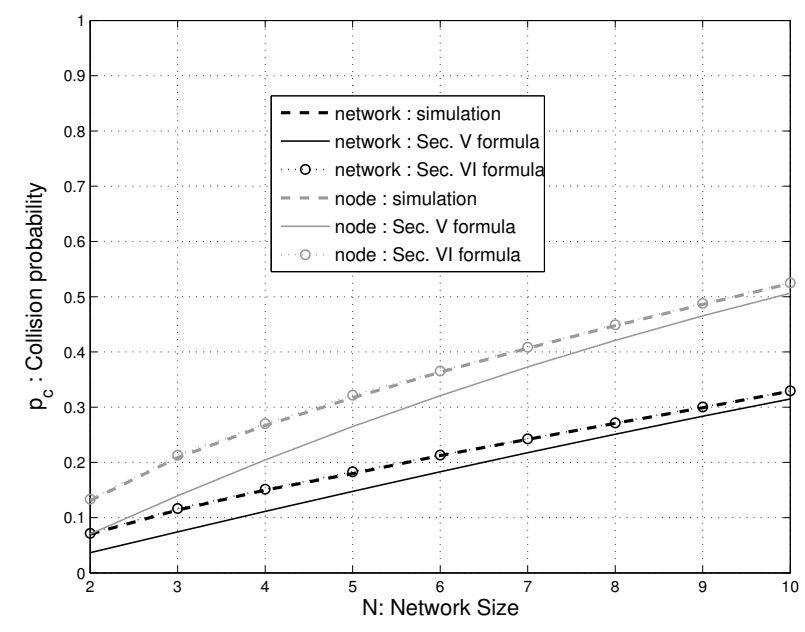

Fig. 9. Comparison between simulation and formula for $p_{\mathrm{c}_{*}}$ and $p_{\mathrm{c}^{\circ}}$

\section{Packet Discard Probability}

As can be seen in Fig. 10, $p_{d}$ as given in (37) exhibits a large error of $78 \%$ for two-node network, which gradually reduces to about $5 \%$ for a 9-node network. To interpret this discrepancy, using (32), (33), and (35), we have

$$
\begin{aligned}
p_{d}= & \operatorname{Pr}\left\{A_{1} \cdots A_{R+1}: \mathrm{COL}\right\} \\
& +\sum_{i=0}^{R} \operatorname{Pr}\left\{A_{1} \cdots A_{i}: \operatorname{COL} \cap A_{i+1}: \text { FAIL }\right\} .
\end{aligned}
$$

In order to derive (37), we used A1 and A2. A1 affects the value of $p_{\text {FAIL }}$ and turns out to be the one that contributes the most to the difference between simulation and analytical result.
Without $\mathrm{A} 1$, the exact value of $p_{\text {FAIL }}$ is

$$
p_{\text {FAIL }}=\prod_{i=0}^{M}\left(1-y_{i}\right)
$$

where $y_{i}=\left(1-\alpha_{i}\right)\left(1-\beta_{i}\right)$.

As of $p_{\mathrm{CoL}}$, even though its value depends on the attempt number, which means that $\mathrm{A} 2$ is not exactly true for $p_{\mathrm{COL}}$ itself, it turns out that $\operatorname{Pr}\left\{A_{1} \cdots A_{i}: \mathrm{COL}\right\} \simeq p_{\mathrm{COL}}^{i}$. Therefore, in this section, we can continue to use $\mathrm{A} 2$ without any significant impact on the results.

The value of $p_{d}$ can therefore be calculated using (37), but with $p_{\text {FAIL }}$ obtained from (51).

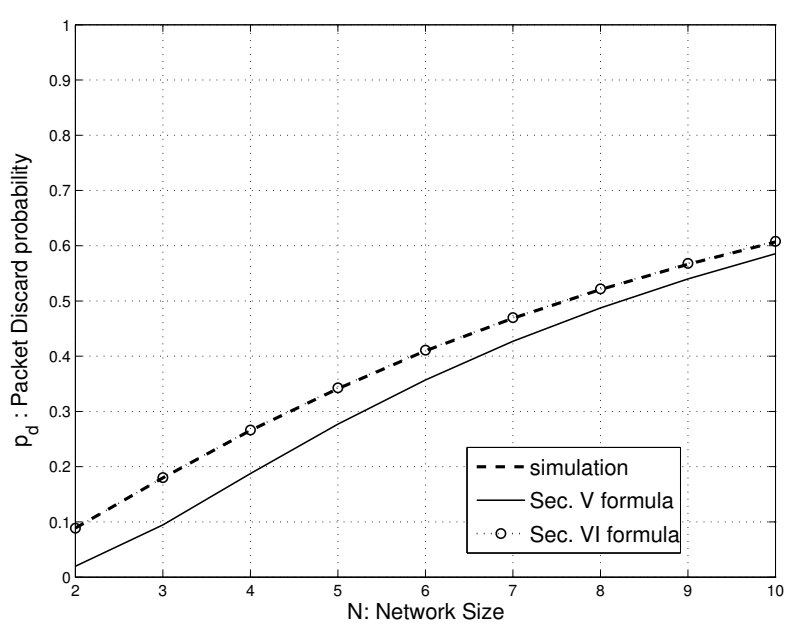

Fig. 10. Comparison between simulation and formula for $p_{d}$.

\section{E. Power Consumption}

The average power consumption formulation of (38), in Section V-E, was derived without using A1-A5. However, as can be seen in Fig. 11, there is a small difference between the simulation and the analytical results. This is mainly due to A1 used in computing $p_{\text {FAIL }}$. The transmission and ACK states are the states in which the most energy is spent, and they are reached only when a transmission attempt does not end in FAIL; thus, the probability that an attempt ends in FAIL, $p_{\text {FAIL }}$, has a key role in (38). As explained in Section VI-D, the exact $p_{\text {FAIL }}$ is given by (51), where $\alpha_{i}$ and $\beta_{i}$ are taken into account.

In order to obtain the correct power consumption, the exact $p_{\text {FAIL }}$ has to be replaced in all its occurrences in (38) including in the $\bar{n}_{B}$ and $\bar{n}_{C}$ formulas, (57) and (61), whose values are strongly affected by $p_{\text {FALL }}$. The power consumption resulted from this replacement is also depicted in Fig. 11.

\section{F. Delay}

Similarly to the case of average power consumption, in (39) we derived the average delay without using any assumptions. However, we did use some of the assumptions in order to compute the final formulation of the parameters involved (i.e., $\bar{n}_{B_{t x}}, \bar{n}_{C_{t x}}$, and $\left.\bar{r}_{s u c}\right)$. In detail, $\bar{n}_{B_{t x}}$ and $\bar{n}_{C_{t x}}$ are affected by $\mathrm{A} 1$, which turns out to be one of the main reasons of the 


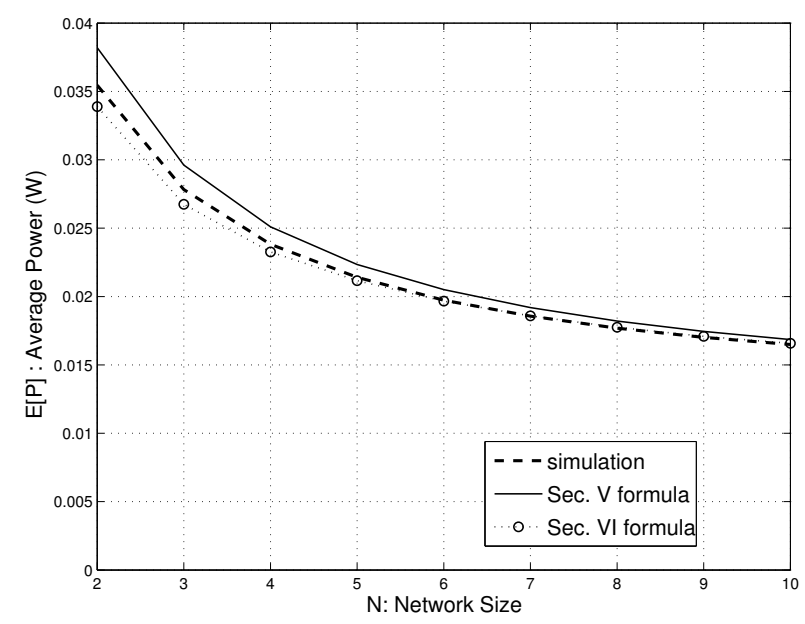

Fig. 11. Average Power Consumption, $\bar{P}$, with the power level for different operating modes given by $P_{t x}=80.7 \mathrm{~mW}, P_{r x}=80.1 \mathrm{~mW}$, and $P_{i d}=1.5$ $\mu \mathrm{W}$ from CC2430 transceiver data sheet.

difference between simulation and analytical results for the average delay. The exact $\bar{n}_{B_{t x}}$ is derived in Appendix C.

The small discrepancy between simulation and formula, shown in Fig. 12, is also partly due to A2 used in calculating $\bar{r}_{\text {suc }}$. To derive $\bar{r}_{\text {suc }}$ in (42), we assumed that the probability of the $i^{\text {th }}$ attempt ending in success, $p_{\mathrm{SUc}(i)}$, or in collision, $p_{\mathrm{COL}(i)}$, is the same as that for any other attempt, i.e., $p_{\mathrm{SUC}}=p_{\mathrm{SUc}(i)}$, and $p_{\mathrm{CoL}}=p_{\mathrm{CoL}(i)}, \forall i, 1 \leq i \leq R+1$. It turns out that these probabilities actually depend on the attempt number, $i$. Mainly, the probability that the first attempt ends in SUC or COL is different from that for any other attempt. Without A2, the exact $\bar{r}_{\text {suc }}$ is

$$
\begin{aligned}
\bar{r}_{\text {suc }} & =\sum_{i=0}^{R} i \frac{\operatorname{Pr}\left\{A_{1} \cdots A_{i}: \mathrm{COL} \cap A_{i+1}: \mathrm{SUC}\right\}}{\operatorname{Pr}\{\mathrm{pkt} \mathrm{SUC}\}} \\
& =\frac{1}{1-p_{d}} \sum_{i=0}^{R} i p_{\mathrm{SUC}(i+1)} \prod_{j=1}^{i} p_{\mathrm{COL}(j)} \\
& =\frac{1}{1-p_{d}} \sum_{i=0}^{R} i p_{\mathrm{SUC}(i+1)} \times p_{\mathrm{COL}(1 \cdots i)}
\end{aligned}
$$

where $p_{\mathrm{COL}(1 \cdots i)}$ is the probability that all the attempts from the first to the $i^{\text {th }}$ end in collision.

\section{CONCLUSION}

This paper has studied the behavior of key networking metrics of IEEE 802.15.4 beacon-enabled nodes.

The assumptions that underlie the Markov chain analyses available to date have been scrutinized and some deficiencies have been pointed out. In particular, it has been shown thatcontrary to what is assumed in those analyses - the probability of sensing the channel free can vary widely from one backoff stage to another. Likewise, it has been shown that-again contrary to prior assumptions - the probability of sensing the channel free is not independent of the number of nodes that are sensing the channel. The implications of these assumptions have been discussed, and their impact on the characterization

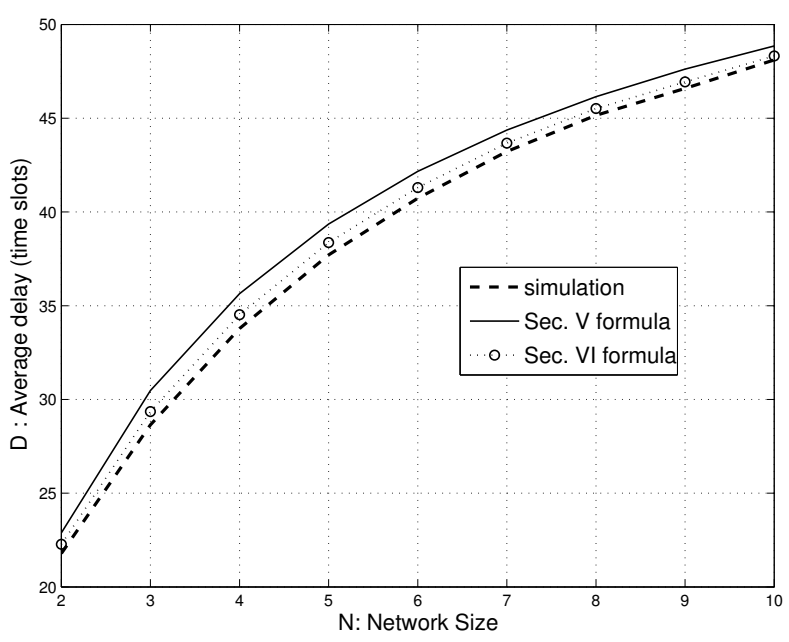

Fig. 12. Comparison between simulation and formula for $\bar{D}$.

of the various networking metrics has been assessed. Circumventing these assumptions, accurate results are obtained at the expense of a more computationally intensive approach (part analytical, part simulation).

Besides examining the aforementioned assumptions, this paper has also extended the model and the performance evaluation to encompass retransmissions, and it has further corrected imprecise uses of the Markov chain approach.

As a by-product of the work, a comprehensive event-driven simulator has been assembled and made freely available to the research community.

\section{ACKNOWLEDGMENT}

This work was partially supported by the Projects CONSOLIDER-INGENIO 2010 CSD2008-00010 "COMONSENS" and TEC2009-13000.

\section{APPENDIX}

\section{A. A Lemma on Conditional Probability}

Lemma 1: If $B_{i}$ are disjoint and symmetric, such that $\operatorname{Pr}\left\{A \mid B_{i}\right\}=\operatorname{Pr}\left\{A \mid B_{j}\right\}$, and $\operatorname{Pr}\left\{B_{i}\right\}=\operatorname{Pr}\left\{B_{j}\right\}, \forall i, j \in$ $\{1 \cdots N\}$, then

$$
\operatorname{Pr}\left\{A \mid \cup B_{i}\right\}=\operatorname{Pr}\left\{A \mid B_{i}\right\}
$$

Proof:

$$
\begin{aligned}
& \operatorname{Pr}\left\{A \mid \cup_{i=1}^{N} B_{i}\right\}=\frac{\sum_{i=1}^{N} \operatorname{Pr}\left\{A \mid B_{i}\right\} \operatorname{Pr}\left\{B_{i}\right\}}{\sum_{i=1}^{N} \operatorname{Pr}\left\{B_{i}\right\}} \\
& =\frac{N \operatorname{Pr}\left\{A \mid B_{i}\right\} \operatorname{Pr}\left\{B_{i}\right\}}{N \operatorname{Pr}\left\{B_{i}\right\}}=\operatorname{Pr}\left\{A \mid B_{i}\right\}
\end{aligned}
$$

B. Mean Number of Backoffs and CCAs Using the Markov Chain Simplifying Assumptions

To calculate the average delay and the average power consumption, we need to know how many slots on average a node spends per attempt performing backoff and CCA. 
1) Mean Number of Backoffs: For an attempt to fail, it needs to unsuccessfully go through all the $M+1$ backoff stages, whereas an attempt that ends in a transmission can end after a successful sensing in any backoff stage. Based on this,

$$
\bar{n}_{B_{t x}}=\sum_{i=0}^{M}\left(\sum_{k=0}^{i} \frac{W_{k}-1}{2}\right) \frac{p_{S_{i}}}{1-p_{\text {FAIL }}}
$$

where $p_{S_{i}}$ is the probability that the first time two consecutive CCAs are successful is in backoff stage $i$, given by

$$
p_{S_{i}}=y(1-y)^{i}, \quad 0 \leq i \leq M .
$$

In turn,

$$
\bar{n}_{B_{f}}=\sum_{k=0}^{M}\left(\frac{W_{k}-1}{2}\right) \frac{p_{S_{M}}}{p_{\mathrm{FAlL}}}=\sum_{k=0}^{M} \frac{W_{k}-1}{2}
$$

Finally, the mean number of slots that a node spends per attempt in backoff is

$$
\bar{n}_{B}=\bar{n}_{B_{t x}}\left(1-p_{\text {FAIL }}\right)+\bar{n}_{B_{f}} p_{\text {FAIL }} \cdot
$$

2) Mean Number of CCAs: For an attempt to end in transmission, a node needs to succeed in the access procedure in some backoff stage. If the access procedure succeeds in stage $i$, it means that it had two successful CCAs in that stage, and at least one failed CCA in stages 0 to $i-1$. In other words, it must have had $k$ successful $\mathrm{CCA}_{1}$ 's and failed $\mathrm{CCA}_{2}$ 's, for some $k \leq i$, and $i-k$ failed $\mathrm{CCA}_{1}$ 's. This happens with probability

$$
p_{f_{i, k}}=\left(\begin{array}{c}
i \\
k
\end{array}\right)[(1-\alpha) \beta]^{k} \cdot \alpha^{i-k} .
$$

In this case, there will be a total of $i+k$ CCAs performed during the failed accesses plus an additional two successful CCAs at stage $i$ where the access procedure succeeds. Thus, the mean number of CCAs before a successful access procedure is given by

$$
\begin{aligned}
\bar{n}_{C_{t x}} & =y \sum_{i=0}^{M} \sum_{k=0}^{i}(i+k+2) \frac{p_{f_{i, k}}}{1-p_{\text {FAIL }}} \\
& =2+[2(1-y)-\alpha]\left[\frac{1}{y}-(M+1) \frac{(1-y)^{M}}{1-p_{\text {FAIL }}}\right] .
\end{aligned}
$$

In the case of an access procedure failure, there are $M+1$ failed attempts and no successful consecutive CCAs. Thus, the mean number of CCAs due to an access failure is

$$
\bar{n}_{C_{f}}=\sum_{k=0}^{M+1}(M+1+k) \frac{p_{f_{M+1, k}}}{p_{\mathrm{FALL}}}=(M+1)\left(2-\frac{\alpha}{1-y}\right) .
$$

Finally, the mean number of CCAs per attempt is

$$
\bar{n}_{C}=\bar{n}_{C_{t x}}\left(1-p_{\mathrm{FALL}}\right)+\bar{n}_{C_{f}} p_{\mathrm{FAlL}} .
$$

\section{Mean number of Backoffs and CCAs in Section VI}

In this appendix, we discuss the effect of A1-A5 on the mean number of backoff and CCAs. We do not depict the simulation results due to space limitations. Similar results are depicted in [13] for the no-ACK mode.
1) Mean Number of Backoffs: In Appendix B we used A1 to compute $\bar{n}_{B_{t x}}$. Without invoking this assumption, the exact $\bar{n}_{B_{t x}}$ is still given by (54), but $p_{S_{i}}$ has to be calculated using

$$
p_{S_{i}}= \begin{cases}y_{0}, & i=0, \\ y_{i} \prod_{k=0}^{i-1}\left(1-y_{k}\right), & 1 \leq i \leq M\end{cases}
$$

where $y_{i}=\left(1-\alpha_{i}\right)\left(1-\beta_{i}\right)$, and $p_{\text {FAL }}$ has to be computed according to (51).

The mean number of slots spent in backoff before an access procedure failure, $\bar{n}_{B_{f}}$, given by (56), is not a function of $\alpha$ and $\beta$ and, therefore, it is valid independently of A1. Moreover, $\bar{n}_{B_{f}}$ is not a function of $N$ because every discarded packet goes through all the backoff stages and, hence, the number of slots spent in backoff for a discarded packet only depends on the random backoff value drawn at every backoff stage. Under A3, for a given backoff stage $i$, an average of $\left(W_{i}-1\right) / 2$ slots are spent in backoff before performing the CCA. As it turns out, the value obtained from (56) is very close but always slightly lower than the one obtained from simulation. This is because $\alpha$ is not completely independent of the random backoff value drawn. In fact, the packets that end up being discarded are those that draw a larger backoff value.

2) Mean Number of CCAs: For $\bar{n}_{C_{t x}}$, as for $\bar{n}_{B_{t x}}$, there is a difference (although smaller both in relative and absolute terms) between simulation and formula. We conjecture that this difference is also due to A1. However, we will not validate this conjecture here because the calculation of $\bar{n}_{C_{t x}}$ as a function of $\alpha_{i}$ and $\beta_{i}$ becomes so complex as to obscure any possible insight and is not warranted given the very small differences.

\section{Event-Driven Network Simulator}

In order to validate the analytical results, a large-scale event-driven simulator has been implemented in Matlab. The network is simulated under the same conditions of the analytical model, i.e., in the ACK mode, with retransmissions, and disregarding the superframe structure. For the simulation, no assumptions are made on the dependence of the nodes or backoff stages and transmission attempts and, thus, the simulation truly reflects the behavior of the network under the aforementioned conditions, and not the behavior of the Markov chain model.

For the MAC parameters, the default values defined by the standard (i.e., macMinBE $=3$, macMaxBE $=5, M=4$, and $R=3$ ) are used and the packet length is fixed for all nodes to $L=7$ slots. For illustrative purposes, when computing the power consumption the parameter values specified for Chipcon 802.15.4-compliant RF transceiver CC2430 [14] are utilized. The simulation results have been obtained over $10^{8}$ slots.

The simulator can be downloaded from http://www.dtic.upf. edu/ alozano/software.

\section{REFERENCES}

[1] Zigbee Alliance, "Zigbee," http://www.zigbee.org. 
[2] IEEE std. 802.15.4, Part. 15.4: Wireless Medium Access Control (MAC) and Physical Layer (PHY) Specifications for Low-Rate Wireless Personal Area Networks (LR-WPANs), IEEE standard for Information Technology, IEEE-SA Standards Board, Sept. 2006.

[3] A. Bachir, M. Dohler, T. Watteyne, and K.K. Leung, "MAC Essentials for Wireless Sensor Networks," IEEE Communications Surveys and Tutorials, to appear.

[4] G. Bianchi, "Performance Analysis of the IEEE 802.11 Distributed Cooridination Function," IEEE Journal on Selected Areas in Communications, vol. 18, no. 3, pp. 535-547, 2000.

[5] J. Mišić, S. Shafi, and V. B. Mišić, "The Impact of MAC Parameters on the Performance of 802.15.4 PAN," Ad Hoc Networks, vol. 3, no. 5, pp. 509-528, Sept. 2005.

[6] J. Mišić and V. B. Mišić, "Access Delay for Nodes with Finite Buffers in IEEE 802.15.4 Beacon Enabled PAN with Uplink Transmissions," Computer Communications, vol. 28, no. 10, Jun. 2005.

[7] S. Pollin, M. Ergen, S. C. Ergen, B. Bougard, L. Van der Perre, I. Moermann, A. Bahai, P. Varaiya, and F. Catthoor, "Performance Analysis of Slotted Carrier Sense IEEE 802.15.4 Medium Access Layer," IEEE Transactions on Wireless Communications, vol. 7, no. 9, Sept. 2008.

[8] P.K. Sahoo and J. Sheu, "Modeling IEEE 802.15.4 based Wireless Sensor Network with packet retry limits," in Proc. of the 5th ACM Symposium on Performance Evaluation of Wireless Ad Hoc, Sensor, and Ubiquitous Networks, Canada, Oct. 2008.

[9] R. K. Patro, M. Raina, V. Ganapathy, M. Shamaiah, and C. Thejaswi, "Analysis and improvement of contention access protocol in IEEE 802.15.4 star network," in Proc of IEEE Internatonal Conference on Mobile Adhoc and Sensor Systems (MASS'07), 2007.

[10] H. Wen, C. Lin, Z.J. Chen, H. Yin, T. He, and E. Dutkiewicz, "An Improved Markov Model for IEEE 802.15. 4 Slotted CSMA/CA Mechanism," Journal of Computer Science and Technology, vol. 24, no. 3, pp. 495-504, 2009.

[11] C.Y. Jung, H.Y.Hwang, D.K.Sung, and G.U.Hwang, "Enhanced Markov Chain Model and Throughput Analysis of the Slotted CSMA/CA for IEEE 802.15.4 Under Unsaturated Traffic Conditions," IEEE Transactions on Vehicular Technology, vol. 58, no. 1, Jan. 2009.

[12] IEEE std. 802.11, Information Technology - Telecommun. and Information Exchange between Systems. Local and Metropolitan Area Networks. Specific Requirements. Part 11: Wireless LAN MAC and PHY Specifications, ANSI/IEEE Std. 802.11, ISO/IEC 8802-11, 1st edition, 1999.

[13] M.R. Palattella, A. Faridi, G. Boggia, P. Camarda, L.A. Grieco, M. Dohler, and A. Lozano, Performance Analysis of the IEEE 802.15.4 MAC Layer, 2010.

[14] Chipcon Products from Texas Instruments, CC2430 Preliminary Data Sheet (rev. 2.1) SWRSO36F, Jun. 2007 